Article

\title{
An Application of Symmetry Approach to Finance: Gauge Symmetry in Finance
}

\author{
Shipeng Zhou ${ }^{1, \star}$ and Liuqing Xiao ${ }^{2}$ \\ ${ }^{1}$ Business School, University of Shanghai for Science and Technology, 516 Jungong Road, Shanghai \\ 200093, China \\ ${ }^{2}$ Department of Mathematics, Shanghai Jiao Tong University, 800 Dongchuan Road, Shanghai 200240, \\ China; E-Mail: lucyxiao917@yahoo.com.cn
}

* Author to whom correspondence should be addressed; E-Mail: Shipengzhou@yahoo.com.cn.

Received: 19 September 2010 / Accepted: 17 October 2010 / Published: 21 October 2010

\begin{abstract}
The paper presents an application of symmetry approach to finance. This symmetry approach comes from the gauge field theory in Physics. We revise the pricing model of financial derivatives in a financial market in a gauge symmetry view, and rewrite it as a partial differential equation on a fiber bundle in covariant differential form so as to have invariance in form. The paper shows the form of the pricing equation can keep invariant under all the local numéraire transformations, this symmetry behind the pricing equation of derivatives is revealed. In addition a corresponding relationship between the curvature of the fiber bundle and the arbitrage in finance arises.
\end{abstract}

Keywords: options pricing equation; gauge symmetry; fiber bundle

Classification: PACS 89.65.Gh, 11.15.-q

\section{Introduction}

The dynamics of the prices of securities and derivatives in finance market are complicated phenomena, but the pricing problem of derivatives is resolved by some basic principles. A famous pricing equation of financial derivatives is the Black-Scholes equation, which is determined by a partial differential equation according to the principle of arbitrage-free equilibrium. This equation is exquisite in mathematics and plays an important role in pricing financial derivatives. We need to ask whether behind the basic principle 
there is some symmetry? In the paper we try to argue that the equation should have some symmetry, i.e., the form of the equation be invariant under a set of specified transformations. We ponder over the problem whether this equation depends upon the units of prices of the assets. If the equation is fundamental in financial theory, the equation should have some covariance, that is, the form of the equation keeps invariant under change of the units. In finance the transformations may be the change of numéraire or measure unit of asset values, because the asset values have only a relative meaning but not an absolute one. Then this symmetry ought to be considered. We show the existence of this symmetry in the paper, which gives a geometrical insight into the pricing models of financial derivatives.

We call the change of numéraire or measure unit of asset values gauge transformation or numéraire transformation hereinafter and we refer to this symmetry of pricing equations under the set of the gauge transformations as a gauge symmetry. In fact the gauge transformations are to multiply the values of assets or the price functions of derivatives by a factor (multiplier) like money exchange in finance so that they have a comprehensive financial meaning. The price function of derivatives is a function of the prices of the underlying assets and time. Furthermore, all the possible prices of the underlying assets compose a vector space which is called basis price space and all the possible prices of derivatives compose a vector space which is called pricing space. We construct a fiber bundle: its base space is the basis price space and its standard fiber is the pricing space, and its structure group consists of gauge transformations. We lift the price function of options defined on the basis price space to a section on the fiber bundle in order to introduce a geometrical formwork for the pricing model of derivatives and to consider symmetry under the gauge transformations. The gauge symmetry plays an important role in our approach. We try to think of it as a fundamental principle in the analysis of derivative pricing just like theoretical physics.

The idea of gauge symmetry in finance is due to K. Ilinski [1] and K. Young [2]. Young posed symmetry under numéraire transformations in financial market and Ilinski's main work was deducing the Black-Scholes equation of options from the action minimization of a Lagrangian. Their works are creative and instructive, but unfortunately this kind of study is pursued by few of researchers in finance and Econophysics. In the paper we take a different way. Our research way is not minimization of action but covariance of pricing equations under numéraire transformations, which have some explicit financial meaning (also see [3]).

\section{Basic Assumption and Pricing Equations of Options}

We consider only the pricing problem of European-style options with multiple underlying assets in this paper, but it is possible to extend this approach in the paper to deal with more general financial derivatives or contingent claims. As is well known, the value of options depends upon the price dynamics of the underlying assets, which are stochastic processes. We suppose that in a financial market there are $n$ tradable risky underlying assets, which may have correlation among themselves, and one risk-free asset such as a bond. All the assets are called basis assets.

We denote the prices of all basis assets in the market by a vector $\boldsymbol{x}=\left(x^{i}\right)=\left(x^{0}, x^{1}, \cdots, x^{n}\right)^{T}$ which is called basis price vector, where the first component $x^{0}$ of the vector represents the price of the risk-free bond. A basis price space consists of a set of all feasible basis price vectors. Let the basis price space be the positive cone of an $(n+1)$-dimensional Euclidean space $\mathcal{P}=\mathbb{R}_{++}^{n+1}$ and let the time axis be $\mathbb{R}$. Then we define a manifold $\mathcal{M}=\mathcal{P} \times \mathbb{R}$, which is simply called basis price-time space. 
We assume that the price fluctuation of each risky asset is driven by a Brownian motion-random source, the dynamics of these prices are described by the following Itô processes [4]:

$$
\mathrm{d} x^{i}=a^{i}(\boldsymbol{x}, t) \mathrm{d} t+b^{i}(\boldsymbol{x}, t) \mathrm{d} W^{i}, \quad i=1, \cdots, n
$$

where the coefficients $a^{i}$ and $b^{i}$ represent the drift and volatility of the $i$-th asset respectively and $W^{i}$ is a Brownian motion. The correlation among the risky assets is described by

$$
\mathrm{d} W^{i} \mathrm{~d} W^{j}=\rho^{i j}(\boldsymbol{x}, t) \mathrm{d} t, \quad i, j=1, \cdots, n
$$

where $\rho^{i j}$ characterizes the covariance between $\mathrm{d} W^{i}$ and $\mathrm{d} W^{j}$ (also it is just their correlation coefficient, and $\rho^{i j}=1, \forall i=j$ ) (see [5]). We may assume the covariance matrix $\boldsymbol{\rho}=\left(\rho^{i j}\right)_{n \times n}$ is strictly positive definite. In general the matrix is not constant and it relates to a metric tensor to be defined in the basis price space.

The dynamics of the bond price is a deterministic process as follows:

$$
\mathrm{d} x^{0}=r x^{0} \mathrm{~d} t, \quad x^{0}(0)>0
$$

where $r$ is the risk-free interest rate in the market (note 'risk-free' means $r$ is constant or a deterministic function of time $t$ ). The bond can play the role of value reference in the market, i.e., it often serves as a reference numéraire such as the unit of cash currency in the market. Of course more generally the interest rate could be stochastic. It is dominated by the following random process:

$$
\mathrm{d} x^{0}=a^{0}(\boldsymbol{x}, t) x^{0} \mathrm{~d} t+\sum_{k=1}^{n} b_{k}^{0}(\boldsymbol{x}, t) x^{0} \mathrm{~d} W^{k}
$$

Note this process includes no new random sources.

As in the treatment in most literature we assume the dynamics of asset prices are the following Itô Processes (see [4])

$$
\begin{array}{lc}
\mathrm{d} x^{i}=\mu^{i}(\boldsymbol{x}, t) x^{i} \mathrm{~d} t+\sigma^{i}(\boldsymbol{x}, t) x^{i} \mathrm{~d} W^{i}, & i=1, \cdots, n \\
\mathrm{~d} W^{i} \mathrm{~d} W^{j}=\rho^{i j}(\boldsymbol{x}, t) \mathrm{d} t, & i, j=1, \cdots, n
\end{array}
$$

where $\mu^{i}$ and $\sigma^{i}$ are the drift and volatility of the $i$-th asset return respectively.

According to Dimensional Analysis we assert that $\mu^{i}$ and $\sigma^{i}$ are quantities without units. Then both $\mu^{i}$ and $\sigma^{i}$ are homogeneous functions of degree 0 for the variables $x^{0}, x^{1}, \cdots, x^{n}$, that is, the functions $\mu^{i}$ and $\sigma^{i}$ can be rewritten as a function of a set of variables which consist of only dimensionless ratios between two asset prices such as $x^{k} / x^{l}$.

Suppose the market is complete and has no friction. Consider the pricing problem of an European-style option with multiple assets. The price (evaluation) of the option at the time $t$ can be written as $V=V(\boldsymbol{x}, t)$, i.e., it is a function of the prices of all the underlying assets $\boldsymbol{x}$ and the time $t$, when $0 \leq t \leq T$, where $T$ is the expired time of this option contract.

Each component $x^{i}$ in the price vector $\boldsymbol{x}$ and the price $V$ of the option have the same unit of cash currency. It is not difficult to prove the following theorem using the Buckingham's $\Pi$ theorem of Dimensional Analysis [6]. 
Theorem 1. $V(\boldsymbol{x}, t)$ can be written as

$$
V(\boldsymbol{x}, t)=\sum_{i=0}^{n} \phi^{i}(\boldsymbol{x}, t) x^{i}
$$

where every $\phi^{i}(\boldsymbol{x}, t)$ is dimensionless and $V(\boldsymbol{x}, t)$ is a homogeneous function of degree 1 for the variables $\boldsymbol{x}$.

Proof. The Buckingham's $\Pi$ theorem tells us: because for every variable $x_{i}$ the function $V / x_{i}$ is dimensionless, $V$ can be written as the product of a function of dimensionless variables and the variable $x_{i}$. Hence generally $V$ can be written as the sum of all such products.

On the other hand, by the Euler theorem for the homogeneous functions of degree 1, we have

$$
V(\boldsymbol{x}, t)=\sum_{i=0}^{n} \frac{\partial V(\boldsymbol{x}, t)}{\partial x^{i}} x^{i}=\sum_{i=0}^{n} \partial_{i} V x^{i}
$$

where we set $\partial_{i}=\partial / \partial x^{i}$. So the identities $\phi^{i}=\partial_{i} V$ hold for all $i$. The expression (7) or (8) is seen as a portfolio of investment in which $\phi^{i}$ denotes a portion of the $i$-th asset, this is a dimensionless quantity.

By Itô's formula we have

$$
\mathrm{d} V=\left(\partial_{t} V+\frac{1}{2} \sum_{i, j=1}^{n} \rho^{i j} \sigma^{i} \sigma^{j} x^{i} x^{j} \partial_{i} \partial_{j} V\right) \mathrm{d} t+\sum_{i=0}^{n} \partial_{i} V \mathrm{~d} x^{i}
$$

Note that the subscripts $i$ and $j$ of the summation of the second term in the above expression start from 1. Therefore, if $V(\boldsymbol{x}, t)$ satisfies the following equation:

$$
\partial_{t} V+\frac{1}{2} \sum_{i, j=1}^{n} \rho^{i j} \sigma^{i} \sigma^{j} x^{i} x^{j} \partial_{i} \partial_{j} V=0
$$

this portfolio of investment is just self-financing because the expressions $\mathrm{d} V=\sum_{i=0}^{n} \phi_{i} \mathrm{~d} x^{i}$ and $V=\sum_{i=0}^{n} \phi_{i} x^{i}$ hold together. Let $g(\boldsymbol{x})$ be the value function at the expire date of the option. When the boundary condition $V(\boldsymbol{x}, T)=g(\boldsymbol{x})$ is satisfied, this self-financing portfolio duplicates the cash flow of the European-style option. Thus $V(\boldsymbol{x}, t)$ is the price of the option at the time $t$, the Equation (10) becomes the pricing equation for the option according to the principle of arbitrage-free equilibrium.

The Equation (10) is a linear parabolic partial differential equation of second order, which has an elliptic operator: $\mathcal{L}=\frac{1}{2} \sum_{i, j=1}^{n} \rho^{i j} \sigma^{i} \sigma^{j} x^{i} x^{j} \partial_{i} \partial_{j}$. Then the Equation (10) is written briefly as a operator form: $\left(\partial_{t}+\mathcal{L}\right) V=0$.

Setting $P(t)=x^{0}(t)$ and $\boldsymbol{S}=\left(s_{i}\right)=\boldsymbol{x}(0 \leq t \leq T)$. When the interest rate $r$ is constant, $P(t)=e^{-r(T-t)}$. In this case we can rewrite the Equation (10) to the general Black-Scholes equation with multiple assets. In fact we define a value function $f(\boldsymbol{S}, t)$ such that

$$
f(P(t) \boldsymbol{S}, t) / P(t)=V(1, \boldsymbol{S}, t)
$$

By some calculation of partial derivatives we can deduce the general Black-Scholes equation for $f$ :

$$
\partial_{t} f+r \sum_{i=1}^{n} S_{i} \partial_{S_{i}} f+\frac{1}{2} \sum_{i, j=1}^{n} \rho^{i j} \sigma^{i} \sigma^{j} S_{i} S_{j} \partial_{S_{i}} \partial_{S_{j}} f-r f=0
$$

Conversely for the Black-Scholes equation (12) we take the variable substitutions as follows:

$$
y_{i}=\frac{1}{\sigma^{i}}\left(r-\frac{\left(\sigma^{i}\right)^{2}}{2}\right)(T-t)+\frac{1}{\sigma^{i}} \ln \left(S_{i}\right), \quad i=1, \cdots, n
$$


and

$$
\tilde{f}(\boldsymbol{y}, t)=e^{r(T-t)} f
$$

The Equation (12) is simplified to a form of diffusion equations:

$$
\partial_{t} \tilde{f}+\frac{1}{2} \sum_{i, j=1}^{n} \rho^{i j} \partial_{y_{i}} \partial_{y_{j}} \tilde{f}=0
$$

This is a variant form of Equation (10). Hence Equation (10) is seen as a kind of typical form of the pricing equation for European style options and is to be studied in detail below.

\section{Numéraire Transformation}

We consider a numéraire transformation, which is a change of the measure unit of assets. Because the price of an asset in the market can be used to serve as a numéraire, let $z=z(\boldsymbol{x}, t)$ be the price of this asset. Its dynamics is depicted by the following equation:

$$
\mathrm{d} z / z=\gamma(\boldsymbol{x}, t) \mathrm{d} t+\sum_{k=1}^{n} \lambda^{k}(\boldsymbol{x}, t) \mathrm{d} W^{k}
$$

where $\gamma(\boldsymbol{x}, t)$ and $\lambda^{k}(\boldsymbol{x}, t)$ are homogeneous functions of degree 0 for $\boldsymbol{x}$ and $z>0$ holds everywhere. Note that this numéraire transformation is local, that is to say, the transformation is different at every point in the basis price-time space. This transformation acts the price of each asset and the price of the option. It gives: $x^{i} \rightarrow \tilde{x}^{i}=x^{i} / z$ and $V \rightarrow V^{\prime}(\boldsymbol{x}, t)=V(\boldsymbol{x}, t) / z$. Under the transformation those variances and covariances $\sigma^{i}, \rho^{i j} \forall i, j$, are invariant due to their homogeneity of degree 0 . Because $z>0$ everywhere we can write $z(\boldsymbol{x}, t)=e^{\varphi(\boldsymbol{x}, t)}$ by a function $\varphi(\boldsymbol{x}, t)$. Then a numéraire transformation for the option price $V$ can be written as

$$
V \rightarrow V^{\prime}=e^{-\varphi(\boldsymbol{x}, t)} V(\boldsymbol{x}, t)
$$

where the multiplier $e^{-\varphi(\boldsymbol{x}, t)}$ is like a discount factor in finance. This kind of transformation can be seen as a 'generalized discounting'. Such transformation of numéraire is just a so-called gauge transformation, which can be seen as an analog of that in Physics.

In the next section we prove that the pricing Equation (10), of options keeps the same form of equations under the above transformation, that is, the pricing equation has invariance for numéraire. So we refer to it as a covariant form of pricing equation.

\section{Geometric Framework of Pricing Equation and Symmetry}

It is seen that the above model of option pricing has already built on the manifold of the basis price-time space. Furthermore this can be extended to a model built on a fiber bundle, that is to say, the model of option pricing can be geometrized and the above transformations becomes actions of a group for the fibers of option prices. Because of the similarity between the above model and the gauge field models in Physics, the approach of gauge field theory could be naturally applied to analyze the problem of option pricing and so we refer to it as the gauge field modeling for option pricing. 


\subsection{Model of Fiber Bundle for Options}

We now consider the basis price-time space, which is a manifold. Obviously such a manifold is trivial in mathematics, but the financial meaning of introducing this manifold here is: there is a family of (local) neighborhoods which cover the total space of the manifold. The numéraire systems of assets in different local neighborhoods may be different and so a local coordinate system in a neighborhood on the manifold corresponds to a numéraire system in the neighborhood. Moreover the scales of time can also can be different at different points on the manifold and there is a transformation between two local coordinate systems in the intersection of two neighborhoods. These transformations correspond to the transformations of numéraires. In the paper we suppose all the transformations are smooth, i.e., the manifold is smooth.

Because the price of options is a function of vectors in the basis price-time space (except for path-dependent options of which the prices depend upon the paths through which they have passed in the past), that is say, this defines a scalar field in the space and we call it field of pricing. Generally one can consider several options based on the same basis price-time space together so that we can take the group of the prices of these options for a vector. Then all the vectors make up of a vector field in a vector space in which the coordinate basis just represents the numéraire unit. In this paper we only consider the scalar field case, but it is immediate to extend to higher-dimensional vector field case. Because the numéraire is changeable locally, a vector space for the prices of options is equipped at every point $\boldsymbol{x}$ in the basis price-time space, the vector space is called the fiber space or fiber (for short) $\boldsymbol{E}_{x}$ at this point, and every point carries a fiber. Note that different fiber may take a different numéraire of the prices of options so that different fibers may have a different coordinate system (i.e., a frame of the fiber space), that is, the numéraire systems can change from one point to another point over the basis price-time space. This kind of geometric structure just leads to the notion of a fiber bundle (see [7] for detail). Hence the union $\cup_{x \in \mathcal{M}} \boldsymbol{E}_{x}$ of all the disjoint fibers in the basis price-time space forms a fiber bundle, which is denoted by $\mathbb{E}$ or $\pi: \mathbb{E} \rightarrow \mathcal{M}$. Here the basis price-time space, $\mathcal{M}$, is called base space and $\pi$ is a smooth projection map to the base space. All the fibers have a same type of space, which is called standard fiber (space). If the standard fiber is a vector space, we call the fiber bundle a vector bundle; if the standard fiber is a Lie group, we call it a principal bundle. The former is used to represent the prices of options and the latter plays the role of numéraire transformations. Hence it is exactly to say that the field of pricing is a smooth section on the fiber bundle: $s: \mathcal{M} \rightarrow \mathbb{E}$, of course $\pi \circ s=\mathrm{i} d: \mathcal{M} \rightarrow \mathcal{M}$. We denote the set of all the sections on the fiber bundle $\mathbb{E}$ by $\Gamma(\mathbb{E})$.

Now we see that the notion of fiber bundle is a quite natural descriptive framework for the pricing model of options, in which the pricing function of options is a section on the bundle. So the problem of option pricing just is to determine such section on the fiber bundle by some equation in mathematics, which relies on to a differential structure of fiber bundles. This leads us to using covariant differential, the correspondent connection and curvature on the bundle. 


\subsection{Covariant Form of Pricing Equation and Gauge Symmetry}

We go back to consider the pricing equation of an European-style option with a stochastic bond determined by the equation (4). Let $\boldsymbol{x} \in \mathcal{P}$ and

$$
g^{i j}=\frac{1}{2} \rho^{i j} \sigma^{i} \sigma^{j} x^{i} x^{j}, \quad i, j=0,1, \cdots, n
$$

where these $\rho^{0 i}$ and $\sigma^{0}$ are easily given by computing the all covariances between $x^{0}$ and $x^{i}$. Note that $x^{i}>0, \forall i$. The above elements $g^{i j}$ form a strictly positive definite matrix $\left(g^{i j}\right)_{(n+1) \times(n+1)}$. Then the elements of its inverse matrix $\left(g_{i j}\right)_{(n+1) \times(n+1)}$ define a metric tensor in the $(n+1)$-dimensional orientable Riemann space $\Re\left(\mathbb{R}_{++}^{n+1}, \mathcal{G}\right)$ :

$$
\mathcal{G}=\sum_{i, j=0}^{n} g_{i j} \mathrm{~d} x^{i} \otimes \mathrm{d} x^{j}
$$

where its determinant is denoted by $g=\operatorname{det}\left(g_{i j}\right)$. Then the pricing Equation (10) becomes

$$
\partial_{t} V+\sum_{i, j=0}^{n} g^{i j} \partial_{i} \partial_{j} V=\partial_{t} V+\mathcal{L} V=0
$$

Note that in the case of a deterministic bond the Riemann space becomes $n$-dimensional and all the subscripts $i$ and $j$ in the above expressions should start from 1 .

Consider now the problem of covariance of the pricing Equation (20) under the local numéraire transformations as in Physics [7]:

$$
V \rightarrow V^{\prime}=e^{-\varphi(\boldsymbol{x}, t)} V
$$

The form of the equation for $V^{\prime}$ will be different from that for $V$. Of course it stays covariant obviously if the above transformation is global, i.e., if the exponent function $\varphi$ is constant. In order that the form of the equation still keeps covariant under the local numéraire transformations, it needs to do some modification for the form of the equation.

Firstly we ought to change the above equation into an 'extended diffusion equation' form which is built in the above Riemann space and keeps linearity:

$$
\partial_{t} V+\Delta V+B V=0
$$

where $B$ is a multiplier to be determined and $\Delta$ is the Laplace operator in the Riemann space:

$$
\Delta=g^{-\frac{1}{2}} \sum_{i, j=0}^{n} \partial_{i}\left(g^{\frac{1}{2}} g^{i j} \partial_{j}\right)
$$

However, the form of this diffusion Equation (22) is not also covariant under the local numéraire transformations (21).

Secondly we must change the partial derivatives into the covariant derivatives:

$$
\partial_{i} \rightarrow \mathrm{D}_{i}=\partial_{i}+A_{i} \quad \forall i
$$

In the above expression these $A_{i}$ are connection coefficients on the fiber bundle, that is to say, the Laplace operator is replaced by a so-called covariant Laplace-Beltrami operator:

$$
\tilde{\Delta}_{\boldsymbol{x}}=g^{-\frac{1}{2}} \sum_{i, j=0}^{n} \mathrm{D}_{i}\left(g^{\frac{1}{2}} g^{i j} \mathrm{D}_{j}\right)
$$


where the subscript $\boldsymbol{x}$ of $\tilde{\Delta}_{\boldsymbol{x}}$ denotes that the operator $\tilde{\Delta}$ takes partial derivatives only with respect to $\boldsymbol{x}$. At the same time we may introduce a covariant derivative with respect to the time variable as follows:

$$
\mathrm{D}_{t}=\partial_{t}+B
$$

Then Equation (22) is written as a compact expression:

$$
\mathrm{D}_{t} V+\tilde{\Delta}_{\boldsymbol{x}} V=0
$$

This equation is called the Laplace-Beltrami equation.

Finally, in order that the Laplace-Beltrami Equation (27) be covariant, we only require that the covariant derivatives and the connection coefficients satisfy the following covariant properties:

$$
\begin{aligned}
& \mathrm{D}_{i}^{\prime} V^{\prime}=\left(\mathrm{D}_{i} V\right)^{\prime}=e^{-\varphi(\boldsymbol{x}, t)} \mathrm{D}_{i} V \quad \forall i \\
& \mathrm{D}_{t}^{\prime} V^{\prime}=\left(\mathrm{D}_{t} V\right)^{\prime}=e^{-\varphi(\boldsymbol{x}, t)} \mathrm{D}_{t} V
\end{aligned}
$$

that is

$$
\begin{aligned}
\left(\partial_{i}+A^{\prime}{ }_{i}\right)\left(e^{-\varphi(\boldsymbol{x}, t)} V\right) & =e^{-\varphi(\boldsymbol{x}, t)}\left(\partial_{i}+A_{i}\right) V \quad \forall i \\
\left(\partial_{t}+B^{\prime}\right)\left(e^{-\varphi(\boldsymbol{x}, t)} V\right) & =e^{-\varphi(\boldsymbol{x}, t)}\left(\partial_{t}+B\right) V
\end{aligned}
$$

Then for all $i$ and $t$ we must have

$$
\begin{aligned}
A_{i} \rightarrow A^{\prime} & =A_{i}+\partial_{i} \varphi \quad \forall i \\
B & \rightarrow B^{\prime}=B+\partial_{t} \varphi
\end{aligned}
$$

In the fiber bundle $\pi: \mathbb{E} \rightarrow \mathcal{M}$ the structure group consists of all the numéraire transformations acting on the section of option prices on the bundle such as positive multipliers and the transformations are similar to the gauge transformations in Physics, that is

$$
V(\boldsymbol{x}, t) \rightarrow V^{\prime}(\boldsymbol{x}, t)=g(\boldsymbol{x}, t) \cdot V(\boldsymbol{x}, t)
$$

where $g(\boldsymbol{x}, t)$ is an element of the transformation group acting on the fiber $\boldsymbol{E}_{(\boldsymbol{x}, t)}=\pi^{-1}(\boldsymbol{x}, t)$. We omit the action operator ' ', writing simply the action of the group $g \cdot V$ as $g V$. This should satisfy the following properties of transformations:

$$
\mathrm{D}_{i}^{\prime} V^{\prime}=\left(\mathrm{D}_{i} V\right)^{\prime}=g\left(\mathrm{D}_{i} V\right)=g \mathrm{D}_{i}\left(g^{-1} V^{\prime}\right) \quad \forall i
$$

so that

$$
\mathrm{D}_{i}^{\prime}=g \mathrm{D}_{i} g^{-1} \quad \forall i
$$

Then according to Leibniz's rule in a local coordinate expression for all $i$ we have

$$
\begin{aligned}
\left(g \mathrm{D}_{i} g^{-1}\right) V^{\prime} & =\left(g\left(\partial_{i}\left(g^{-1} V^{\prime}\right)+A_{i} g^{-1} V^{\prime}\right)\right) \\
& =\left(\left(\partial_{i}+\left[g\left(\partial_{i} g^{-1}\right)+g A_{i} g^{-1}\right]\right) V^{\prime}\right) \\
& =\mathrm{D}_{i}{ }^{\prime} V^{\prime}=\left(\left(\partial_{i}+A_{i}{ }^{\prime}\right) V^{\prime}\right)
\end{aligned}
$$


Because $V^{\prime}$ is arbitrary, this gives the transformation rule of the connection coefficients under the gauge transformation:

$$
A^{\prime}{ }_{i}=g A_{i} g^{-1}+g\left(\partial_{i} g^{-1}\right) \quad \forall i
$$

Substituting $g(\boldsymbol{x}, t)=e^{-\varphi(\boldsymbol{x}, t)}$ we obtain the expression (29a) easily. We have the expression (29b) similarly.

For the equivalence between Equation (27) and Equation (20), we give the following theorem:

Theorem 2. If the connection coefficients take

$$
A_{i}=-\frac{1}{2} g^{-\frac{1}{2}} \sum_{j=0}^{n}\left(g_{i j} \sum_{k=0}^{n} \partial_{k}\left(g^{\frac{1}{2}} g^{k j}\right)\right) \quad \forall i
$$

and

$$
B=\sum_{i, j=0}^{n} g^{i j}\left(A_{i} A_{j}-\partial_{i} A_{j}\right)
$$

then Equation (27) is equivalent to Equation (20) and is covariant under the local neméraire transformation (21).

Proof. We want to prove $\left(\tilde{\Delta}_{\boldsymbol{x}}+B\right) s=(\Delta f) \boldsymbol{e}$ for any section on the fiber bundle $s=f \boldsymbol{e} \in \Gamma(\mathbb{E})$, where $e$ is the basis vector of the fiber. We do the following computation in a local coordinate expression:

$$
\begin{aligned}
\left(\tilde{\Delta}_{\boldsymbol{x}}+B\right) s & =\left\{g^{-\frac{1}{2}} \sum_{i, j=0}^{n} \mathrm{D}_{i}\left(g^{\frac{1}{2}} g^{i j} \mathrm{D}_{j}\right)+B\right\} f \boldsymbol{e} \\
& =g^{-\frac{1}{2}}\left\{\sum_{i, j=0}^{n} \partial_{i}\left[g^{\frac{1}{2}} g^{i j}\left(\partial_{j} f+A_{j} f\right)\right]\right. \\
& \left.+\sum_{i, j=0}^{n} A_{i} g^{\frac{1}{2}} g^{i j}\left(\partial_{j} f+A_{j} f\right)\right\} \boldsymbol{e}+B f \boldsymbol{e}
\end{aligned}
$$

Then we obtain

$$
\begin{aligned}
\left(\tilde{\Delta}_{x}+B\right) s= & \sum_{i, j=0}^{n}\left\{g^{-\frac{1}{2}}\left(\partial_{i}\left(g^{\frac{1}{2}} g^{i j} \partial_{j} f\right)+\partial_{i}\left(g^{\frac{1}{2}} g^{i j} A_{j} f\right)\right)+g^{i j} \partial_{i}\left(\partial_{j} f\right)\right. \\
& \left.+g^{i j}\left(\partial_{i} A_{j}\right) f\right\} \boldsymbol{e}+\sum_{i, j=0}^{n}\left(A_{i} g^{i j} \partial_{i} f+A_{i} g^{i j} A_{j} f\right) \boldsymbol{e}+B f \boldsymbol{e} \\
= & \sum_{i, j=0}^{n} g^{i j} \partial_{i}\left(\partial_{j} f\right) \boldsymbol{e}+\sum_{i, j=0}^{n}\left[g^{-\frac{1}{2}} \partial_{i}\left(g^{\frac{1}{2}} g^{i j}\right)+2 g^{i j} A_{i}\right] \partial_{j} f \boldsymbol{e} \\
& +\left\{\sum_{i, j=0}^{n}\left[g^{-\frac{1}{2}} \partial_{i}\left(g^{\frac{1}{2}} g^{i j}\right) A_{j}+g^{i j} \partial_{i} A_{j}+A_{i} g^{i j} A_{j}\right]+B\right\} f \boldsymbol{e}
\end{aligned}
$$

Above we use the symmetry: $g^{i j}=g^{j i} \forall i, j$ for the second term in the last identity. Because of the arbitrariness of $f$ the equivalence between Equations (27) and (20) holds if an only if the following conditions are satisfied:

$$
\sum_{i=0}^{n}\left[g^{-\frac{1}{2}} \partial_{i}\left(g^{\frac{1}{2}} g^{i j}\right)+2 g^{i j} A_{j}\right]=0 \quad \forall j
$$

and

$$
\sum_{i, j=0}^{n}\left[g^{-\frac{1}{2}} \partial_{i}\left(g^{\frac{1}{2}} g^{i j}\right) A_{j}+g^{i j} \partial_{i} A_{j}+A_{i} g^{i j} A_{j}\right]+B=0
$$

This proves the expressions (32) and (33).

Now we prove the covariance of the Equation (27). In fact according to the covariant properties (28) and (31) we have

$$
\begin{aligned}
e^{-\varphi(\boldsymbol{x}, t)} \mathrm{D}_{t} V & =\mathrm{D}_{t}\left(e^{-\varphi(\boldsymbol{x}, t)} V\right)-\left(\partial_{t} e^{-\varphi(\boldsymbol{x}, t)}\right) V \\
& =\mathrm{D}_{t}\left(e^{-\varphi(\boldsymbol{x}, t)} V\right)+\partial_{t} \varphi\left(e^{-\varphi(\boldsymbol{x}, t)} V\right) \\
& =\left(\mathrm{D}_{t}+\partial_{t} \varphi\right)\left(e^{-\varphi(\boldsymbol{x}, t)} V\right)=\mathrm{D}_{t}^{\prime} V^{\prime}
\end{aligned}
$$


and

$$
\begin{aligned}
e^{-\varphi(\boldsymbol{x}, t)} \tilde{\Delta}_{\boldsymbol{x}} V & =e^{-\varphi(\boldsymbol{x}, t)} g^{-\frac{1}{2}} \sum_{i, j=0}^{n} \mathrm{D}_{i}\left(g^{\frac{1}{2}} g^{i j} \mathrm{D}_{j}\right) V \\
& =g^{-\frac{1}{2}} \sum_{i, j=0}^{n} \mathrm{D}_{i}\left(g^{\frac{1}{2}} g^{i j} \mathrm{D}_{j}\right)\left(e^{-\varphi(\boldsymbol{x}, t)} V\right) \\
& =\tilde{\Delta}_{\boldsymbol{x}}^{\prime} V^{\prime}
\end{aligned}
$$

Multiplying the both sides of the Equation (27) by $e^{-\varphi(x, t)}$ from the left we get immediately

$$
\mathrm{D}_{t}^{\prime} V^{\prime}+\tilde{\Delta}_{x}^{\prime} V^{\prime}=0
$$

This proves the covariance of the Equation (27).

\section{Connection and Curvature}

Now we obtain the covariant form of the pricing equation of options. Here the covariant derivatives are crucial and the covariant derivatives are determined by a specific connection. The connection may be also written as a differential 1-form:

$$
A=\sum_{i=0}^{n} A_{i} \mathrm{~d} x^{i}
$$

It is called a gauge field in Physics. The covariance of the equation is referred to as its gauge symmetry, which is a kind of invariance under transformations. It reflects a geometric characteristics of the option pricing.

We know that the connection of a fiber bundle determines the curvature of the fiber bundle. The curvature of a fiber bundle in a local coordinate is defined as

$$
F_{i j}=\partial_{i} A_{j}-\partial_{j} A_{i}+\left[A_{i}, A_{j}\right] \quad \forall i, j
$$

where $[\cdot, \cdot]$ is the Lie bracket. (Note that this Lie bracket term in Equation above vanishes for a vector bundle with fibers of one dimension, but this term is often indispensable for a vector bundle with fibers of multiple dimensions. Although this paper only deals with the former, some extension for the latter is not impossible.) If there exists a smooth function $\chi(\boldsymbol{x}, t)$ such that $A_{i}=\partial_{i} \chi \forall i$, i.e., the differential 1-form is exact: $A=\mathrm{d} \chi$, where $\mathrm{d}$ is exterior differential. Then evidently this connection has $\mathrm{d} A=0$ and the curvature tensor of the bundle is null, $F_{i j}=0 \forall i, j$. It means that the bundle is a flat space. If $B=\partial_{t} \chi$ also holds at the same time, then the above covariant pricing equation of options (27) reverts to a typical partial differential equation with a Laplace operator in Riemann space $\partial_{t} V+\Delta V=0$ after the transformation $V \rightarrow V^{\prime}=e^{-\chi(\boldsymbol{x}, t)} V$.

The following example of an option with two assets demonstrates the procedure of the symmetry (covariance) analysis above.

Suppose there be two risky assets and one risk-free bond in a financial market, i.e., $n=2$, and the interest rate $r(t)$ is deterministic. In the dynamics of the risky assets described by the equations (5) all the parameters, $\mu^{i}, \sigma^{i}, \rho\left(\rho=\rho^{12},|\rho|<1\right)$, are constant. We have

$$
\left(g^{i j}\right)=\frac{1}{2}\left(\begin{array}{cc}
\left(\sigma^{1} x^{1}\right)^{2} & \rho \sigma^{1} \sigma^{2} x^{1} x^{2} \\
\rho \sigma^{1} \sigma^{2} x^{1} x^{2} & \left(\sigma^{2} x^{2}\right)^{2}
\end{array}\right)
$$


where the subscripts $i$ and $j$ start from 1 . Then

$$
\left(g_{i j}\right)=\frac{2}{1-\rho^{2}}\left(\begin{array}{cc}
\left(\sigma^{1} x^{1}\right)^{-2} & -\rho /\left(\sigma^{1} \sigma^{2} x^{1} x^{2}\right) \\
-\rho /\left(\sigma^{1} \sigma^{2} x^{1} x^{2}\right) & \left(\sigma^{2} x^{2}\right)^{-2}
\end{array}\right)
$$

and

$$
g=\frac{4}{\left(1-\rho^{2}\right)\left(\sigma^{1} \sigma^{2} x^{1} x^{2}\right)^{2}}
$$

We easy get by computation

$$
|g|^{-\frac{1}{2}} \sum_{k=1}^{n} \partial_{k}\left(|g|^{\frac{1}{2}} g^{k j}\right)=\frac{1}{2}\left(\sigma^{j}\right)^{2} x^{j}, \quad j=1,2
$$

Then

$$
\begin{aligned}
A_{i} & =-\frac{1}{2} \sum_{j=1}^{n} g_{i j}\left(|g|^{-\frac{1}{2}} \sum_{k=1}^{n} \partial_{k}\left(|g|^{\frac{1}{2}} g^{k j}\right)\right) \\
& =-\frac{1}{2\left(1-\rho^{2}\right)}\left(1-\rho \frac{\sigma^{(n+1-i)}}{\sigma^{i}}\right) \frac{1}{x^{i}}, \quad i=1,2 \\
B & =\sum_{i, j=0}^{n} g^{i j}\left(A_{i} A_{j}-\partial_{i} A_{j}\right) \\
& =-\frac{1}{8\left(1-\rho^{2}\right)}\left[\left(\sigma^{1}\right)^{2}+\left(\sigma^{2}\right)^{2}-2 \rho \sigma^{1} \sigma^{2}\right]
\end{aligned}
$$

Hence we take

$$
\begin{aligned}
\chi(\mathbf{x}, t)= & -\frac{1}{2\left(1-\rho^{2}\right)} \sum_{i=1}^{n}\left(1-\rho \frac{\sigma^{(n+1-i)}}{\sigma^{i}}\right) \ln \left(x^{i}\right) \\
& -\frac{1}{8\left(1-\rho^{2}\right)}\left[\left(\sigma^{1}\right)^{2}+\left(\sigma^{2}\right)^{2}-2 \rho \sigma^{1} \sigma^{2}\right] t
\end{aligned}
$$

We have $A=\sum_{i=1}^{n} A_{i} \mathrm{~d} x^{i}=(\mathrm{d} \chi)_{\left(x^{1}, x^{2}\right)}$ and $B=\partial_{t} \chi$. Then the 1-form $A$ is exact and the fiber bundle is flat.

Taking $\varphi=\chi$ and under the transformation $V \rightarrow V^{\prime}=e^{-\varphi(x, t)} V$ we obtain the typical equation in the Riemann space of basis price-time:

$$
\partial_{t} V+\Delta V=0
$$

In fact the result that the fiber bundle is flat in the case of geometric Brownian motion is expected and explicable, because we think that non vanishing curvature on the fiber bundle represents an arbitrage opportunity in the market just like a 'force' in a physical field. In the case of the standard geometric Brownian motions this 'force' vanishes, which means no arbitrage in the market. So we see a correspondent relationship between the geometrical curvature in the fiber bundle and arbitrage-free equilibrium in finance. However, in general the dynamics of the prices of underlying assets are complicated stochastic processes, which are not Brownian motions, and in addition the market could be also incomplete; then there is some arbitrage opportunity on the market. Then the minimization of arbitrage is applied to the pricing problem of options. Hence it is potentially useful for studying further the geometrizing model of options. 


\section{Unification of Covariant Derivatives}

To unify the covariant derivatives with respect to the variables of prices and time in the base space, we modify the base space $\Re\left(\mathbb{R}_{++}^{n+1}, \mathcal{G}\right)$ such that the time space $\mathbb{R}$ is really enclosed into a pseudoriemannian space. We do it by means of enlarging the dimension of the space. We add two new variables: one variable is the time variable and another variable is a dummy variable. Then the dimension of the space becomes $(n+3)$-dimensional.

We denote the $(n+3)$-dimensional pseudoriemannian space by $\tilde{\mathcal{M}}=\Re\left(\mathbb{R}_{++}^{n+1} \times \mathbb{R} \times \mathbb{R}, \mathcal{G}\right)$ and in the space a point is $\tilde{\boldsymbol{x}}=\left(x^{0}, x^{1}, \cdots, x^{n}, x^{n+1}, x^{n+2}\right) \in \tilde{\mathcal{M}}^{T}$, where the $(n+2)$-th variable denotes the time variable and the $(n+3)$-th variable is dummy. We still denote the vector $\tilde{\boldsymbol{x}}$ by $\boldsymbol{x}$ for simplicity. The metric tensor $\mathcal{G}$ of this pseudoriemannian space is defined by the following formula:

$$
\mathcal{G}=\sum_{i, j=0}^{n} g_{i j} \mathrm{~d} x^{i} \otimes \mathrm{d} x^{j}+2 \mathrm{~d} x^{n+1} \otimes \mathrm{d} x^{n+2}+2 \mathrm{~d} x^{n+2} \otimes \mathrm{d} x^{n+1}
$$

Obviously the previous Riemann space $\Re\left(\mathbb{R}_{++}^{n+1}, \mathcal{G}\right)$ can be embedded into this space $\tilde{\mathcal{M}}$. The value of the determinant of the metric matrix $\tilde{g}$ differs from that of $g$ by a factor -4 . Because any non zero constant factor do not influence the definition of the covariant Laplace operator, so the pricing equation is not also influenced.

In the same way we could construct a fiber bundle $\mathbb{E}$, the base manifold of which just is the pseudoriemannian space $\tilde{\mathcal{M}}$ and the fibers are still the same as the previous ones. Its structure group is the set of the strictly positive diagonal matrices with ordinary matrix multiplication, which is denoted by $\Lambda(k)$, where $k$ is the dimension of the fiber space. In the case of $k=1$ we deal with only the group of the positive multipliers: $\Lambda(1)$.

Let $A_{n+1}=B$. Then the covariant derivative of time in the operator (25) is also defined uniformly. Let $A_{n+2}=0$. Because any element $g(\boldsymbol{x}, t)$ in $\Lambda(1)$ does not include the component $x^{n+2}$, it follows that $A_{n+2} \equiv 0$ holds. We have the following results as in Theorem (2):

Theorem 3. Let the covariant Laplace-Beltrami operator on the fiber bundle $\tilde{\mathbb{E}}$ be

$$
\tilde{\Delta}_{\tilde{\mathbb{E}}}=|\tilde{g}|^{-\frac{1}{2}} \sum_{i, j=0}^{n+2} \mathrm{D}_{i}\left(|\tilde{g}|^{\frac{1}{2}} g^{i j} \mathrm{D}_{j}\right)
$$

If the connection coefficients in the above operator take

$$
\begin{aligned}
& A_{i}=-\frac{1}{2} g^{-\frac{1}{2}} \sum_{j=0}^{n}\left(g_{i j} \sum_{k=0}^{n} \partial_{k}\left(g^{\frac{1}{2}} g^{k j}\right)\right), \quad i=0, \cdots, n \\
& A_{n+1}=\sum_{i, j=0}^{n} g^{i j}\left(A_{i} A_{j}-\partial_{i} A_{j}\right) \\
& A_{n+2}=0
\end{aligned}
$$

let $\tilde{V}=V \cdot x^{n+2}$. Then the pricing Equation (20) becomes the following covariant equation:

$$
\tilde{\Delta}_{\tilde{\mathbb{E}}} \tilde{V}=0
$$

Proof. This proof is completely similar to the procedure of the proof of Theorem (2) and it is not difficult to prove that these connection coefficients given in Theorem (3) obey indubitably the transformation rule of connection coefficients under the gauge transformations. 
Thus it can be concluded that the pricing equation becomes a type of covariant Laplace-Beltrami equation with gauge symmetry, which is just like the previous equation other than being more compact in form.

\section{Conclusions}

We apply the theory of gauge fields in Physics to the pricing model of options in finance in this paper. Our work shows that the pricing equation of options can be written as the covariant Laplace equation on a fiber bundle. The symmetry behind the pricing equation of options is revealed. In addition the covariant derivatives with respect to the time variable and the covariant derivatives with respect to the price variable are written in the same way on the fiber bundle by means of the method of enlarging the dimension of the base space. The geometrization of the pricing model of options relates to gauge symmetry, connection, curvature and more uniform form of the pricing equation. The introduction of this approach is natural and the potential extension of the approach is possible. We hope to extend this method into more general option pricing problems in the subsequent research. We expect this method could uncover some implicit and intrinsic properties of the pricing problem of financial derivatives to some extent.

\section{Acknowledgements}

The work is supported by Shanghai Leading Academic Discipline Project under Project No. S30501. We acknowledge the suggestions of Professor Hongan Che and discussions with him.

\section{References and Notes}

1. Ilinski, K. Physics of Finance: Gauge Modeling in Non-equilibrium Pricing; John Wiley and Sons Ltd.: New York, NY, USA, 2001.

2. Young, K. Foreign Exchange Market as a Lattice Gauge Theory. Am. J. Phys. 1999, 67, 862-868.

3. Henry-Labordère P. made a very similar research in the part of geometric framework, see his paper: Solvable local and stochastic volatility models: supersymmetric methods in option pricing, SSRN 2005, available at SSRN: http://ssrn.com/abstract=773568. His geometric framework is about the backward Kolmogorov equation of the conditional probability density for options and is used to making the asymptotic expansion for his model.

4. Etheridge, A. A Course in Financial Calculus; Cambridge University Press: Cambridge, UK, 2002.

5. In fact, we have $\rho^{i j}=\lim _{h \rightarrow 0} \mathbb{E}\left\{\frac{1}{h}\left(W^{i}(t+h)-W^{i}(t)\right)\left(W^{j}(t+h)-W^{j}(t)\right) \mid \mathcal{F}_{t}\right\}$.

6. de Jong, F.J. Dimensional Analysis for Economists; North-Holland: Amsterdam, The Netherlands, 1967.

7. Frankel, T. The Geometry of Physics: An Introduction, 2nd ed.; Cambridge University Press: Cambridge, UK, 2004.

(c) 2010 by the authors; licensee MDPI, Basel, Switzerland. This article is an open access article distributed under the terms and conditions of the Creative Commons Attribution license (http://creativecommons.org/licenses/by/3.0/.) 